\title{
Diagnostic Value of Determination Blast Cell Population Lineage Using WPC Scattergram Hematology Analyzer
}

\author{
Nina Ratnasari ${ }^{1}$, Arifoel Hajat ${ }^{1}$, S. Ugroseno Yudho Bintoro ${ }^{2}$ \\ ${ }^{1}$ Department of Clinical Pathology, Faculty of Medicine, Airlangga University/Dr. Soetomo Academic General Hospital Surabaya, \\ Indonesia.E-mail:nina83ratnasari@gmail.com \\ 2 Department of Internal Medicine, Faculty of Medicine, Airlangga University/Dr. Soetomo Academic General Hospital Surabaya, Indonesia.
}

\begin{abstract}
The diagnosis of hematology malignancies requires examination that includes morphology, immunophenotyping, and cytogenetics. Immunophenotyping is the most trusted examination in determining hematology malignancies lineage, but it is only available in large hospitals and the costs are relatively expensive, so the determination of lineage depends on Bone Marrow Aspiration (BMA) examination. Therefore it is necessary to have an easier and more reliable alternative to assist BMA morphology. White Precursor Cell (WPC) scattergram Sysmex XN-1000 has the capability to differentiate malignancy lineage. The purpose of this study was to determine the diagnostic value of determining lineage generated by WPC scattergram compared to the lineage from BMA examination. Bone marrow aspiration blood samples were simultaneously examined by BMA morphology interpretation using microscope and WPC scattergram Sysmex XN-1000 examination. The hematology malignancies lineage resulting from BMA and WPC scattergram examination was then analyzed statistically to determine the suitability, sensitivity, and specificity. The results of determining the lineage of blast cell population based on WPC scattergram resulted in a suitability with a sensitivity of $93.75 \%$ and specificity of $94.74 \%$ for determining the hematological malignancy of myeloid lineage and $94.74 \%$ and $93.75 \%$ for lymphoid lineage, with a diagnostic accuracy of $94.91 \%$. Based on this study it can be concluded that the WPC scattergram can determine the lineage of hematological malignancies with a suitability and high diagnostic value of lineage based on BMA morphology.
\end{abstract}

Keywords: White precursor cell scattergram, blast cell population, bone marrow aspiration, myeloid, lymphoid, lineage

\section{INTRODUCTION}

Hematology malignancies are an increase in blast cell population resulting from abnormal proliferation of leukocytes in the blood and bone marrow. The incidence of leukemia in the United States is 12.8 per 100,000 people each year. ${ }^{1}$ Leukemia is classified into two subtypes, namely lymphoid and myeloid. These two subtypes are further divided into acute types consisting of Acute Myeloblastic Leukemia (AML) and Acute Lymphoblastic Leukemia (ALL), while the chronic types consist of Chronic Myeloid Leukemia (CML) and Chronic Lymphocytic Leukemia (CLL). ${ }^{2}$

The gold standard in determining diagnosis of leukemia according to the WHO criteria was based on morphology, immunophenotyping, and cytogenetic examination. ${ }^{3}$ Morphology interpretation of Bone Marrow Aspiration (BMA) is a standard examination in determining the diagnosis of hematology malignancies. ${ }^{3,4}$ This examination is quite simple, has a reliable diagnostic value, and is cost-effective, but has a weakness that is the safe invasive procedure, requires well trained expert in the procedure to aspirate and interpreting BMA blood smear. ${ }^{5,6}$ Immunophenotyping is a reliable examination to confirm the diagnosis of hematology malignancies lineage determination, but the cost is quite expensive, it requires special expertise and is only available in a number of health facilities, so other alternative examinations are needed which are easier, relatively inexpensive, reliable, and available in many laboratories, without reducing the diagnostic value.?

Hematology analyzer is now equipped with the latest parameter known as White Precursor Cell (WPC) which can be read on WPC scattergram. Besides WPC scattergram can determine the existence of a blast cell population, it can also help to determine the lineage of the blast cell population. Lymphoblast population or malignant abnormal lymphocytes produce fluorescence with a high intensity compared to other leukocyte cells, whereas myeloblast population (blast) issues the lowest fluorescence intensity so that in WPC SFL-SSC scattergram myeloblast population occupies a low 
position (blast area) and the lymphoblast population occupies a high fluorescence intensity (abnormal lymphocyte area). Myeloblast population has the largest size with less complex cell structure so that it occupies the top left position in the WPC FSC-SSC scattergram while lymphoblast has a small size and is less complex so it occupies the lower left position (abnormal lymphocyte area). ${ }^{8}$ The results of the WPC scattergram examination are said to be myeloid lineage if a cluster is found in the blast area, namely the lower area of WPC SFL-FSC and in the WPC FSC-SSC upper area, and it is said to be a lymphoid lineage if the cluster is in an abnormal lymphocyte position, i.e. the upper area of the WPC SFL-SSC and the lower area of WPC FSC-SSC scattergram. Blast cell area in WPC scattergram are gating automatically by the hematology analyzer.

The determination of lineage based on the WPC scattergram has not been studied widely. The purpose of this study was to determine the suitability of the lineage produced by WPC scattergram against the lineage generated based on BMA morphology.

\section{METHODS}

This was an observational analytical research using a cross-sectional design study. The research was conducted from June to September 2019 at the Clinical Pathology Laboratory of the Dr. Soetomo Hospital Surabaya, collaborating with the Departments of Clinical Pathology and Internal Medicine, Faculty of Medicine Airlangga University/Dr. Soetomo Hospital Surabaya. The study population comprised all patients with an increase of blast cell percentage requested for BMA examinations at the Clinical Pathology Laboratory of the Dr. Soetomo Hospital Surabaya and who signed an informed consent willingness as a study participant of 113 samples. The inclusion criteria of this research was, an increase in blast cell population in adult patients and children resulting from the morphological interpretation of BMA blood smear, while the exclusion criteria was when there was a clot in the BMA blood sample, and no fragments or matrices were found in the BMA blood smear.

The research samples were bone marrow blood aspirations of patients with an increase in blast cell population in adults and children. BMA blood samples were put into EDTA tubes, with a minimum volume of $1 \mathrm{~mL}$, then the samples were examined immediately by hematology analyzer. The BMA blood samples were examined at the same time for morphological interpretation from BMA smear and WPC scattergram Sysmex XN-1000 examination, the results of the examination were interpreted by at least two hematology experts. The hematological malignancy lineage obtained from the morphological readings of BMA and WPC scattergram was subsequently analyzed statistically to determine its suitability, sensitivity, and specificity. The statistical analysis used diagnostic test methods by $2 \times 2$ tables. Gold standard used in this research was the interpretation result of morphology BMA blood smear. This research was approved by the Health Research Ethics Committee of the Dr. Soetomo Hospital, Surabaya.

Research permission was obtained from the Health Research Ethics Committee of the Dr. Soetomo Hospital, Surabaya, with number 1202/KEPK/V/2019.

\section{RESULTS AND DISCUSSION}

The research samples consisted of 113 samples, comprising 64 males (56.6\%), 49 females (43.4\%), with a median value of age 44 years. The age of the patients varied considerably with an age range of 0.020 to 95 years as shown in Table 1 . This research in general only determined the lineage, which in general was not influenced by therapy, clinical diagnosis, so data about it were not collected. The most important factor from this research was the increasing number of blast cell population.

Based on the data in Table 2, the examination results of BMA blood samples showed that the number of WBC patients had a median value of $106.350 \times 10^{3} / \mu \mathrm{L}$ which were counted by hematology analyzer. Other general characteristics known were the presence of NRBC, Immature Granulocyte (IG) and abnormal lymphoid (Abn I) flagging on examining more blood samples with a percentage of 53.1\% (NRBC), 87.6\% (IG) and 54\% (abnormal I), while for the presence of flagging blasts and atypical lymphocytes (atyl I) it was less with a percentage of only $28.3 \%$ (blasts) and $11.5 \%$ (atypical lymphocytes). This study only determined the suitability of the blast cell population lineage based

Table 1. Gender and age of samples

\begin{tabular}{lcr}
\hline \multirow{2}{*}{ Patients Characteristics } & \multicolumn{2}{c}{ Results } \\
\cline { 2 - 3 } & Numbers (n) & $\%$ \\
\hline Gender & 64 & 56.6 \\
$\quad$ Male & 49 & 43.4 \\
$\quad$ Female & \multicolumn{2}{|}{44} \\
Age (year) & \multicolumn{2}{c}{44} \\
$\quad$ Median & \multicolumn{2}{c}{$020-95$} \\
Range & \multicolumn{2}{c}{0.020} \\
\hline
\end{tabular}


Table 2. BMA blood samples characteristics

\begin{tabular}{lcc}
\hline \multirow{2}{*}{ Blood Sample } & \multicolumn{2}{c}{ Results } \\
\cline { 2 - 3 } Characteristics & Numbers (n) & \% \\
\hline WBC & \multicolumn{2}{c}{106.350} \\
$\quad$ Median & \multicolumn{2}{c}{$4.990-984.490$} \\
$\quad$ Range & \multicolumn{2}{c}{} \\
Lineage characteristics & & \\
$\quad$ NRBC & 60 & 53.1 \\
$\quad$ Positive & 53 & 46.9 \\
$\quad$ Negative & & \\
IG (immature granulocyte) & 99 & 87.6 \\
$\quad$ Positive & 14 & 12.4 \\
$\quad$ Negative & & \\
Blast & 32 & 28.3 \\
$\quad$ Positive & 81 & 71.1 \\
$\quad$ Negative & & \\
AbN L (abnormal lymphoid) & & \\
$\quad$ Positive & 61 & 54.0 \\
$\quad$ Negative & 52 & 46.0 \\
Atyl L (atypical lymphoid) & & \\
$\quad$ Positive & 13 & 11.5 \\
$\quad$ Negative & 100 & 88.5 \\
\hline
\end{tabular}

on the results of WPC scattergram, without considering the existence of flagging either from the WPC channel itself or from other channels (WDF channels), which may also support the determination of a lineage.

Based on Table 3, 113 BMA blood samples determined based on morphological characteristics showed that 69 samples (61.1\%) were included as hematology malignancies of myeloid lineage and 44 samples (38.9\%) as lymphoid lineage samples. Myeloid lineage hematology malignancies in this study consisted of $A M L, M D S$, and $C M L$, while lymphoid lineage included ALL, CLL, MM, leukemic phase of Iymphoma and Waldenstrom macroglobulinemia. One hundred and thirteen BMA blood samples were further examined by WPC scattergram and 47 samples (41.6\%) were included into myeloid category, 39 lymphoid samples (34.5\%), and from 27 samples (23.9\%) the lineage could not be concluded with WPC scattergram (inconclusive).

Based on Table 4, forty-five patients with WPC scattergram and morphology of BMA blood smear were included in the myeloid lineage, while lymphoid lineage consisted of 36 patients. Other examination results showed that there were differences in lineage categories between WPC scattergram and morphology of BMA blood smear, two patients with myeloid lineage on WPC scattergram, which should be the lymphoid lineage category on morphology of BMA blood smear, and there were three patients with lymphoid lineage on WPC scattergram, which should be myeloid lineage category on morphology of BMA blood smears.

The results of the WPC scattergram diagnostic test in determining myeloid lineage hematology malignancies yielded a sensitivity value of $93.75 \%$, which meant that the diagnostic test for determining lineage using WPC scattergram could really produce the myeloid lineage category of $93.75 \%$, in other words 93.75\% BMA samples with hematology malignancies of myeloid lineage could be detected using WPC scattergram. The specificity value of the WPC scattergram diagnostic test results obtained at $94.74 \%$ meant that the hematology malignancies of the myeloid lineage could be omitted in $94.74 \%$ of the samples with hematological malignancy of the lymphoid lineage series. The ability of the WPC scattergram diagnostic test in determining the lymphoid lineage series of a hematology malignancies had a sensitivity of $94.74 \%$ and a specificity of $93.75 \%$.

Table 3. Determination lineage based on WPC scattergram

\begin{tabular}{|c|c|c|c|c|c|}
\hline \multirow{2}{*}{ BMA Lineage } & \multicolumn{2}{|c|}{ Result } & \multirow{2}{*}{ WPC Lineage } & \multicolumn{2}{|c|}{ Result } \\
\hline & Numbers (n) & $\%$ & & Numbers (n) & $\%$ \\
\hline $\begin{array}{l}\text { Myeloid } \\
\text { AML } \\
\text { MDS } \\
\text { CML }\end{array}$ & 69 & 61.1 & Myeloid & 47 & 41.6 \\
\hline $\begin{array}{l}\text { Lymphoid } \\
\text { ALL } \\
\text { MM } \\
\text { CLL }\end{array}$ & 44 & 38.9 & Lymphoid & 39 & 34.5 \\
\hline $\begin{array}{l}\text { Leukemic phase of lymphoma and } \\
\text { Waldenstrom macroglobulinemia }\end{array}$ & & & & & \\
\hline & & & Inconclusive & 27 & 23.9 \\
\hline
\end{tabular}


Based on the data in Table 4, it was also known that the Positive Predictive Value (PPV) was 95.74\%, which meant that the proportion of patients with WPC scattergram myeloid lineage series and also including myeloid lineage on BMA examination was 95.74\%. While the Negative Predictive Value (NPV) was $92.31 \%$ which meant that the proportion of patients with WPC scattergram lymphoid lineage and also including lymphoid lineage on BMA examination was $92.31 \%$.

Based on the results of calculations that had been done, it could be concluded in general that the results of diagnostic tests using the WPC scattergram method in determining lineage had an accuracy of $94.91 \%$ included in the high category.

The WPC channel used differences of lipid composition from cell membrane to separate normal and abnormal leukocyte cells so that it could differentiate neoplastic cells from reactive processes. $^{9-11}$ This calculation produced a 2D scattergram, namely: WPC (SFL-SSC) and WPC (FSC-SSC) scattergram. Based on the leukocyte population contained in the WPC scattergram, the hematology malignancies lineage could be concluded as: Myeloid, if a cluster was found in the low WPC SFL-SSC scattergram and high WPC FSC-SSC scattergram areas; Lymphoid, if a cluster was found in the WPC SFL-SSC scattergram high and Iow WPC FSC-SSC scattergram. Scattered areas as shown in Figure 1.

The conclusion of WPC scattergram in 27 samples out of the 113 samples in this study was inconclusive because it did not fulfil the criteria for lymphoid or myeloid lineage in WPC scattergram, so these results were considered as inconclusive. This was likely caused by several factors, including: Only one cluster was found in the high area of WPC SFL-FSC scattergram (single cluster), and no abnormal cluster

Table 4. Diagnostic value of WPC scattergram lineage againts morphology of BMA blood smear in all samples suspected of hematology malignancies

\begin{tabular}{|c|c|c|c|}
\hline \multirow[b]{2}{*}{ WPC } & \multicolumn{2}{|c|}{ BMA } & \multirow[b]{2}{*}{ Total } \\
\hline & $\begin{array}{c}\text { Myeloid } \\
\mathrm{n}\end{array}$ & $\begin{array}{c}\text { Lymphoid } \\
n\end{array}$ & \\
\hline Myeloid & 45 & 2 & 47 \\
\hline Lymphoid & 3 & 36 & 39 \\
\hline Total & 48 & 38 & 86 \\
\hline Sensitivity & $93.75 \%$ & \multicolumn{2}{|c|}{$\mathrm{CI}(83.16-97.85)$} \\
\hline Spesificity & $94.74 \%$ & \multicolumn{2}{|c|}{ CI (82.71 - 98.54) } \\
\hline PPV & $95.74 \%$ & \multicolumn{2}{|c|}{ CI (85.75 - 98.83) } \\
\hline NPV & $92.31 \%$ & \multicolumn{2}{|c|}{ CI (79.68 - 97.35) } \\
\hline Likelihood (+) & 17.81 & \multicolumn{2}{|c|}{ CI $(6.67-47.60)$} \\
\hline Likelihood (-) & 0.07 & \multicolumn{2}{|c|}{$\mathrm{CI}(0.03-0.13)$} \\
\hline Wilcoxon $p$-value & 0.655 & & \\
\hline
\end{tabular}

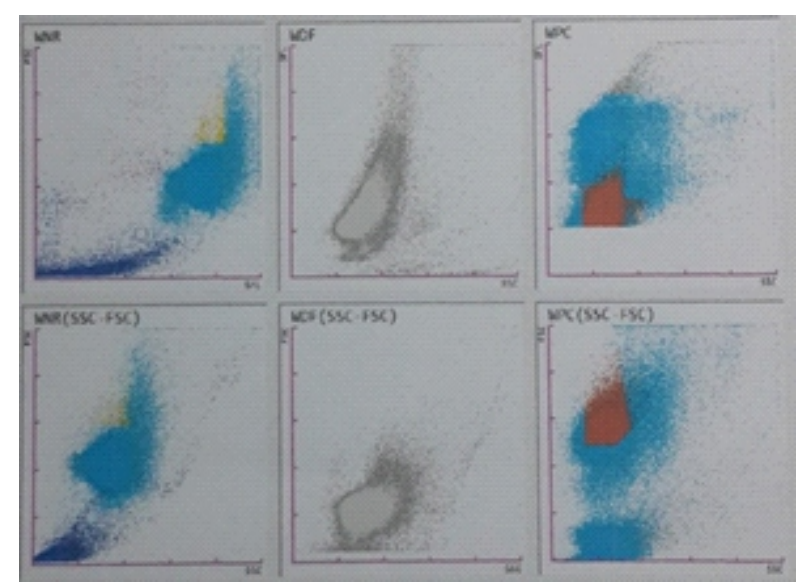

Figure 1. WPC scattergram that showed myeloid lineage

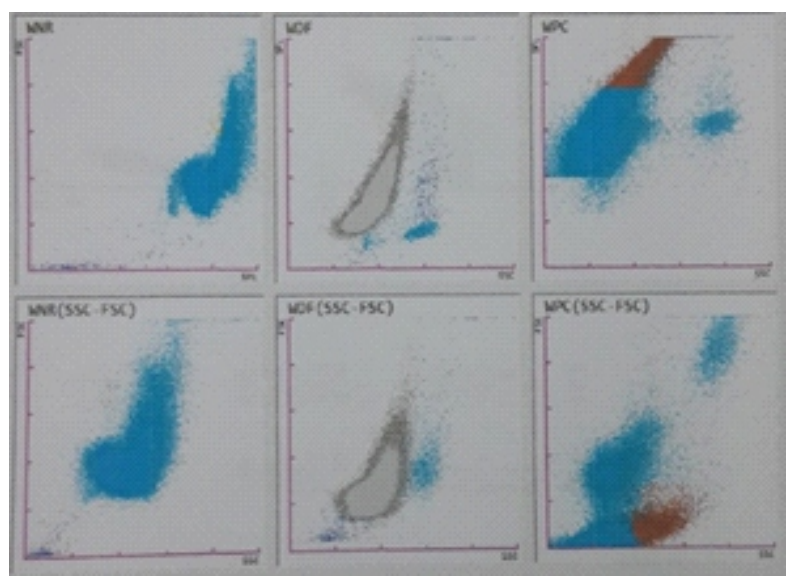

Figure 2. WPC scattergram that showed lymphoid lineage 

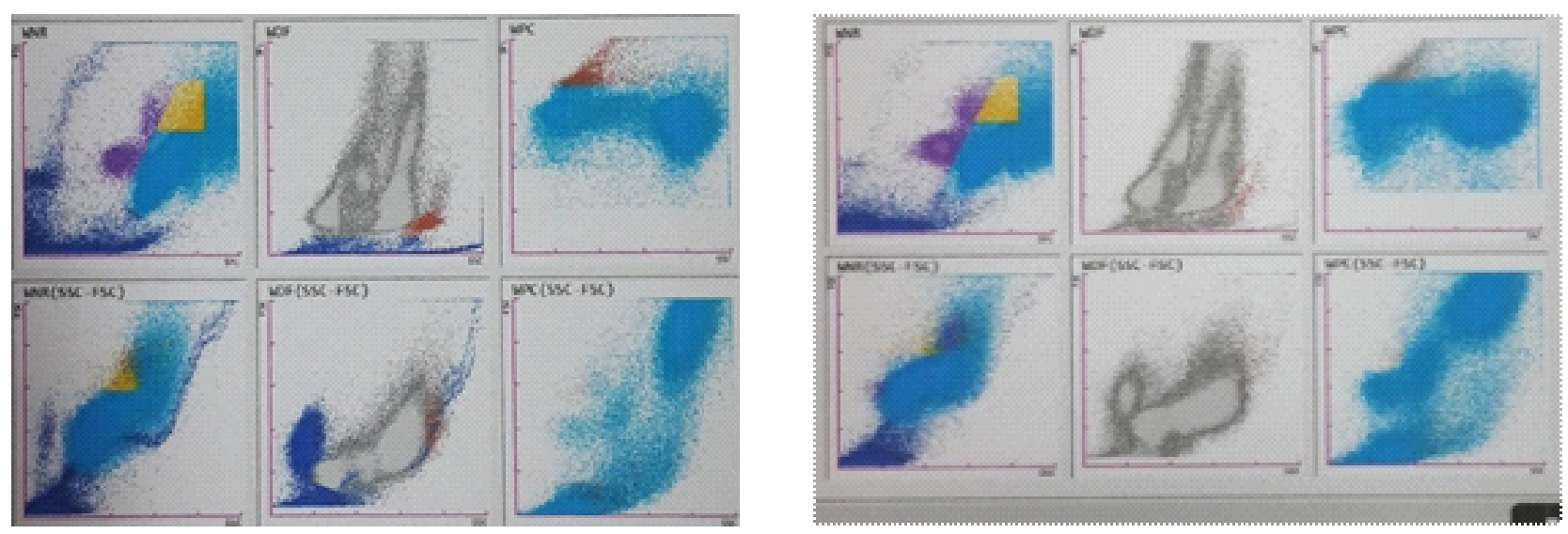

Figure 3a dan 3b. Showing cluster in high area of WPC SFL-SSC scattergram

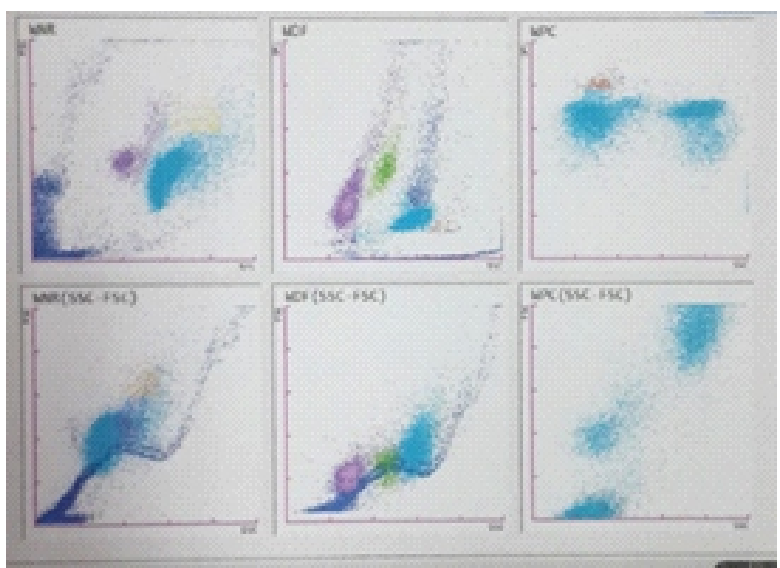

Figure 4. There were no clusters in low and high area in WPC SFL-SSC and WPC FSC-SSC scattergram

was found in WPC FSC-SSC scattergram. The presence of one cluster was likely due to the high percentage of NRBCs and the absence of blast populations or abnormal lymphoid populations as shown in Figure $3 a$ and Figure $3 b$; The number of blast cell population or abnormal lymphoid in WPC scattergram was too low, so it could not form a cluster and was not detected in the low and high WPC SFL-SSC and FSC-SSC scattergram areas as shown in Figure 4; There was a picture of 2 populations (double clusters) in the WPC SFL-SSC area (high and low) and WPC FSC-SSC scattergram (high and low), so it became difficult or could not be concluded as shown in Figure 5.

The WPC scattergram although in some samples yielded inconclusive conclusions but most of them issued conclusive conclusions so that it was very helpful in determining the lineage of a hematology malignancy, while inconclusive results did not generate an error in determining the lineage. Inconclusive results on WPC scattergram WPC were

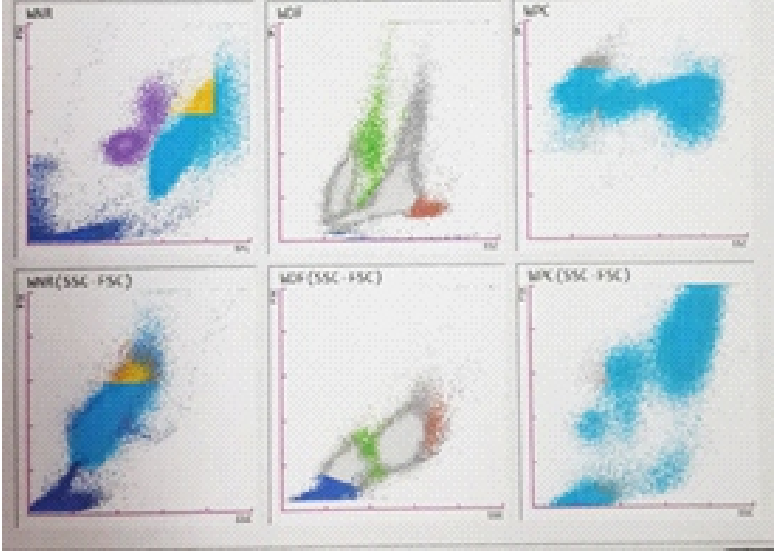

Figure 5. Showing two populastions in high and low WPC SFL-SSC and WPC FSC-SSC scattergram

influenced by interference factors that were likely due to the high percentage of NRBC presentations, thus forming a cluster in a high area, which could be misinterpreted as a high area cluster lymphoid series. Another interference factor was the number of blast cells, which if the number of blast cells was too low, it could not form a cluster, thus the cluster cannot be found in the area which was analyzed.

\section{CONCLUSION AND SUGGESTION}

Based on the results of this study, it could be concluded that the suitability of determination hematology malignancies based on WPC scattegram against lineage generated based on morphological of BMA blood smear yielded a $93.75 \%$ sensitivity and $94.74 \%$ specificity for myeloid lineage, while sensitivity and specificity for lymphoid lineage were $94.74 \%$ and $93.75 \%$. From the sensitivity and specificity results, it could be said that the WPC scattergram could determine the lineage of 
hematologic malignancy with good accuracy with a diagnostic accuracy value of $94.19 \%$.

Not all BMA aspirates in this study were followed by immunophenotyping to confirm the lineage, so that further research is needed regarding WPC scattergram lineage compared with lineage from immunophenotyping.

\section{REFERENCES}

1. Davis AS, Viera AJ, Mead MD. Leukemia: An overview for primary care. American Family Physician, 2014; 89(9): 731-738.

2. Singh $G$, Parmar $P$, Kataria SP, Singh $S$, Sen R. Spectrum of acute and chronic leukemia at a tertiary care hospital, Haryana India, 2016; 4(4): 1115-1118.

3. Shahab F, Raziq F. Clinical presentations of acute leukemia. Journal of the College of Physicians and Surgeon Pakistan, 2014; 24(7): 472-476.

4. Wirawan R. Evaluasi sumsum tulang di Pemeriksaan laboratorium hematologi. Ed Pertama., Jakarta, Badan Penerbit Fakultas Kedokteran Indonesia, 2014; 233-247.

5. Castro WL, Ibanez GB, Perez MAL, Sanchez AS, Jaloma JC, Garcia EM, Penafiel COR. Morphology of leukaemias. Revista Medica Hospital General Mexico, 2016; 79(2): 107-113.
6. Pudasaini S, Prasad KBR, Rauniyar SK, Shrestha R, Gautam K, et al. Interpretation of bone marrow aspiration in hematological disorder. Journal of Pathology of Nepal, 2012; 2: 309-312.

7. Gajendra S. Flow cytometry in acute leukemia. Department of Pathology and Laboratory Medicine, 2016; (1): 1-5.

8. Kawauchi S, Kono M, Takagi Y, Wada A, Morikawa Takashi. The positions of normal leukocytes on the scattergram of the newly developed abnormal cell-detection channel of the XN-Series: A multi-parameter automated hematology analyzers. Sysmex Journal International, 2013; 23(1):1-9.

9. Jones AS, Tailor H, Liesner H, Machin SJ, Briggs CJ. The value of the white precursor cell channel (WPC) on the Sysmex XN-1000 analyser in a specialist Paediatric Hospital. Journal Clinical Pathology, 2015; (68): 161-165.

10. Werner PS, Kohlschein P, Maroz A, Linssen J, Dreibiger K, Burstein C. Performance of the XN-1000 WPC channel-flagging to differentiate reactive and neoplastic leukocytosis. Clinical Chemistry Laboratory Medicine, 2016; 1-9.

11. Sysmex Corporation. White blood cell functionally sensitive assessment of WBC functionality and greater workflow efficiency. Sysmex White Paper White Blood Cell Functionality, 2017 ; 1-7. 